\title{
Auditorías de valor al sistema de gestión de la seguridad y la salud en el trabajo en empresas de transporte terrestre de carga seca*
}

\author{
Value audits of the occupational health and safety management \\ system in land transport companies for dry cargo
}

\section{Auditorías de valor ao sistema de gestão da segurança e saúde no trabalho em transportadoras terrestres de carga seca}

\begin{abstract}
Recibido: 20 de octubre de 2019 Revisado: 10 de febrero de 2020 Aceptado: 5 de mar $\approx$ de 2020

Carlos Alirio Beltrán Rodrígue***

Universidad Santo Tomás - Seccional Tunja

Ángel Leonel Puerto Núñes***

Universidad Distrital Francisco José de Caldas

Harold Wilson Hernánde: Cru****

Universidad Santo Tomás

Cómo citar este artículo: Beltrán-Rodríguez, C. A., Puerto-Núñez A. L., y Hernández-Cruz, H. W. (2020). Auditorías de valor al sistema de gestión de la seguridad y la salud en el trabajo en empresas de transporte terrestre de carga seca. Signos, Investigación en Sistemas de Gestion, 12(2), 59-73. DoI: https://doi.org/10.15332/24631140.5937

* Artículo de resultado de investigación

** Magíster en Ingeniería Industrial, Especialista en Higiene y Salud Ocupacional, Ingeniero Industrial. Grupo de Investigación en Suelo, Planta, Agua y Ambiente (GISPA), Universidad Santo Tomás, Tunja, Colombia. Correo electrónico: dec.industrial@ustatunja.edu.co. ORCID: https://orcid.org/0000-0003-3918-5009.

*** Magíster en Ingeniería Industrial. Ingeniero Industrial. Grupo de Investigación Adquisición y Representación del Conocimiento - Sistemas Expertos y Simulación (ARCO SES), Universidad Distrital Francisco José de Caldas. Bogotá, Colombia. Correo electrónico: productividad.hseq@gmail.com. ORCID: https://orcid.org/0000-0002-9848-0078.

**** Magíster en Ciencias de la Educación, Especialista en Educación Mediada por Tic, Ingeniero Industrial. Universidad Santo Tomás, línea de investigación en calidad y gestión integral. Correo electrónico: harold.hernandez@usantotomas.edu.co. oRCID: https://orcid.org/0000-0002-9374-6703
\end{abstract}




\section{RESUMEN}

Este artículo da una orientación frente al enfoque que debe darse a auditorías internas en el sistema de gestión de seguridad y salud en el trabajo, con el fin de identificar los requisitos que no se establezcan completamente en el Decreto 1072 de 2015. Para ello, se toma como escenario de aplicación un grupo empresarial de Boyacá, conformado por cuatro empresas de transporte de carga. Se estructuró un modelo de gestión que integra las variables que intervienen en un proceso de auditoría de acuerdo con la norma Iso 19011, con el fin de aportar herramientas de evaluación que agreguen valor a sus resultados y a la vez contribuir en los índices de productividad sostenida. Posteriormente, se aplicóla simulación de Forrester, en la que se plantean posibles escenarios y la aplicación de diagramas causales para la interrelación entre las variables endógenas y exógenas.

Palabras clave: auditoría, evaluación de desempeño, sistema de gestión de seguridad y salud en el trabajo, plan estratégico de seguridad vial.

\section{ABSTRACT}

This article provides guidance on the approach of internal audits in the occupational safety and health management system in order to identify requirements not fully provided for in Decree 1072/2015. For this purpose, a Boyacá Business Group, made up of four cargo transport companies, is taken as the application scenario. A management model was created that integrates the variables involved in an audit process in accordance with ISO 19011 in order to provide evaluation tools that add value to their results while contributing to sustained productivity rates. Subsequently, Forrester's simulation was applied, in which possible scenarios and the application of causal diagrams for the interrelationship between endogenous and exogenous variables are proposed.
Keywords: audit, occupational safety and health management system, performance evaluation, strategic road safety plan.

\section{RESUMO}

Este artigo fornece orientações sobre a metodologia que deve ser seguida nas auditorías internas do sistema de gestão da segurança e saúde no trabalho, a fim de identificar requisitos que não estejam completamente relacionados com o Decreto n. ${ }^{0} 1072$ de 2015. Neste sentido, um Grupo Empresarial de Boyacá, na Colômbia, composto por quatro empresas de transporte de carga, é considerado como cenário de aplicação. Foi estruturado um modelo de gestão que integra as variáveis envolvidas nos processos de auditoría de acordo com a norma ISO 19011, a fim de fornecer ferramentas de avaliação que acrescentem valor aos seus resultados e, ao mesmo tempo, contribuam para taxas de produtividade sustentadas. Seguidamente, foi aplicada a simulação de Forrester, na qual são propostos cenários possíveis e a aplicação de diagramas causais para a interrelação entre as variáveis endógenas e exógenas.

Palavras-chave: auditoría, sistema de gestão de segurança e saúde no trabalho, avaliação de desempenho, 0 plano estratégico de segurança viária.

\section{INTRODUCCIÓN}

Los últimos estudios demuestran un alto incremento de accidentalidad vial. Dentro de los factores que influyen en los accidentes de tránsito se encuentra el mecánico, el físico y el humano. El factor mecánico representa el $3 \%$ y está influenciado por la dirección, la suspensión, los frenos, las llantas, los elementos del vehículo. El factor físico corresponde al $3 \%$ y está dado por condiciones de la vía, líquidos en la vía, hundimientos, derrumbes y 
presencia de obstáculos. El factor humano es la causa principal del $94 \%$ de los accidentes, que se generan por imprudencia, negligencia e impericia.

Atendiendo a este contexto y a la exigencia de la Resolución 1565 (Ministerio de Transporte, 2014), en la que se establecen los requisitos del plan estratégico de seguridad vial, se espera que estos lineamientos contribuyan a las organizaciones en el fomento de una cultura de cero accidentes de tránsito y en el desarrollo de las actividades de los colaboradores de una manera segura.

Adicionalmente, todas las organizaciones en Colombia, de acuerdo con lo establecido en el Decreto 1072 (Ministerio del Trabajo, 2015), deben diseñar, implementar, evaluar y mejorar el sistema de gestión de seguridad y salud en el trabajo (SG-SST). Asimismo, este Decreto contempla un requisito de integración con otros sistemas de gestión con los que cuente la organización. En este sentido, se espera que las organizaciones optimicen la gestión y articulen el SG-SST con el plan estratégico de seguridad vial.

El departamento de Boyacá reporta alta accidentalidad y de personas fallecidas a causa de los accidentes de tránsito (Agencia Nacional de Seguridad Vial, 2019); en este sentido, se hace necesario la adopción y cumplimiento de la legislación colombiana sobre el plan estratégico de seguridad vial y las acciones aplicables del SG-SST que aporten en la reducción de la accidentalidad vial por parte de las empresas de la región.

Cabe destacar que Boyacá cuenta con un gran número de empresas del sector industrial, minero y energético, razón por la cual se ve la necesidad de fortalecer el sector transporte y a la vez contar con estrategias de auditoría que permitan evidenciar el cumplimiento de la legislación mencionada, para así contribuir al incremento de la seguridad vial y de la seguridad y salud en el trabajo.

Aunque el Decreto 1072 de 2015 contempla requisitos para el desarrollo de las auditorías internas del SG-SST, las organizaciones no siempre logran tener criterios claros al momento de su realización. Se ha evidenciado que existen diversos criterios al momento de evaluar la eficacia de las acciones de seguridad y salud en el trabajo (Robson et al., 2007) y en los procesos de auditoría de este (Robson, Macdonald, Gray, Van Eerd, \& Bigelow, 2012).

Si bien existen estudios sobre actividades dentro del sector transporte y las auditorías de primera, segunda y tercera parte, no se encuentran estudios de este tipo dirigidos a identificar, evaluar y priorizar condiciones asociadas a procesos de auditoría en SG SST de empresas de transporte. Por eso, en este artículo se presenta una propuesta de auditorías de valor para el SG-SST dirigido principalmente a empresas de transporte terrestre de carga seca.

\section{METODOLOGÍA}

La investigación también contempla un componente descriptivo, ya que permite ordenar el resultado de las observaciones de las conductas, las características, los factores, los procedimientos y otras variables de fenómenos y hechos. Se busca crear un modelo que permita identificar el desempeño y los resultados de procesos de evaluación mediante auditorías de valor que contribuyan en la identificación de oportunidades de mejora del SG-SST.

Los datos fueron recopilados a partir de informes, hallazgos y heurísticas puntuales del proceso de auditoría y de la experiencia de los auditores. Se buscó que, en una primera etapa del desarrollo del proyecto, la evaluación de los hallazgos obtenidos dentro del proceso auditado sea validada y confrontada por el SBC. Todos los datos e información obtenida dentro de la base de datos fueron suministrados por los expertos auditores y los resultados de las auditorías. 
Una vez obtenidos los hechos y las heurísticas de las evaluaciones de los hallazgos en las auditorías, se establecieron las relaciones entre sí. Las relaciones se implementaron por medio de reglas del tipo si una condición, entonces una acción o conclusión. Estas relaciones se ejecutan de acuerdo con el razonamiento que sigue la metodología Forrester. Un ejemplo de una relación es el siguiente: si se evaluó la auditoría, entonces se han generado unos hallazgos; si se generaron los hallazgos, entonces se generan unas acciones.

Como resultado se consideró el análisis de la competencia del auditor a la hora de realizar la auditoría. Su objetivo fue derivar la nueva información de la evaluación de los hallazgos obtenidos en el proceso de ejecución de la auditoría en sus diferentes etapas: planificación, ejecución y elaboración del informe final.

Para dar solución a la problemática de realizar auditorías internas que generen valor del SG-SST en empresas de transporte, tomando como base un grupo empresarial de Boyacá, se consideraron las dimensiones de la metodología Forrester, que considera cuatro aspectos: personas (fisiológicos, psicosociales y de competencias), objetivos (del proceso de auditoría), estrategias (normas y estándares) y tecnologías.

Para ello, se usó la dinámica de sistemas en la que se consideraron diagramas causales que permiten realizar un esquema de la interrelación entre las variables, de manera que se perciba fácilmente la estructura y el comportamiento del sistema. Con ello, se contribuye al mejoramiento de la calidad de vida, la seguridad y salud en el trabajo, y la productividad de los trabajadores de este sector.

Se consideraron como criterios de análisis los factores que influyen en los procesos de auditoría la Iso 19011 y la GTC 45, instrumentos que son dinámicos para la recolección, tratamiento y análisis. Algunos de los factores que pretende analizar esta investigación no están contemplados en dichas normas, para lo cual se deberá ajustar las variables analizadas al mismo protocolo.

De acuerdo con lo establecido en el Decreto 1072 (Ministerio del Trabajo, 2015), se requiere efectuar una auditoría de cumplimiento del SG-SST de forma anual y será planificada con la participación del ComitéParitario o Vigía de Seguridad y Salud en el Trabajo. Además, "el programa de auditoría debe comprender entre otros, la definición de la idoneidad de la persona que sea auditora, el alcance de la auditoría, la periodicidad, la metodología y la presentación de informes, y debe tomarse en consideración resultados de auditorías previas" (Ministerio del Trabajo, 2015, Artículo 2.2.4.6.29, resaltado nuestro).

\section{RESULTADOS Y DISCUSIÓN}

La recolección de datos es un proceso mediante el cual el sujeto, a través de la observación sistemática, y apoyado en un instrumental ad hoc, registra de manera selectiva y codificada los indicadores del estado de las variables con el propósito de hacer referencia a estas y no al objeto, con lo cual se logra resaltar que se trata de un proceso de observación de un área definida conceptualmente y no de un todo indefinido e indiscriminado. Para ello, se elaboró un diagnóstico de los criterios basado en la norma OHSAS 18001:2007 y RUC que incluye factores de riesgo presentes en los lugares de trabajo a través de una encuesta aplicada a una muestra representativa de trabajadores de este sector que nos permita evaluar aspectos ocupacionales y condiciones de trabajo; este se establece mediante matriz de correlación con un resultado de $36 \%$ en avance frente al cumplimiento de requisitos RUC y OHSAS 18001:2007. 
Fiǵura 1. Diagnóstico del SG-STA del grupo empresarial oIT

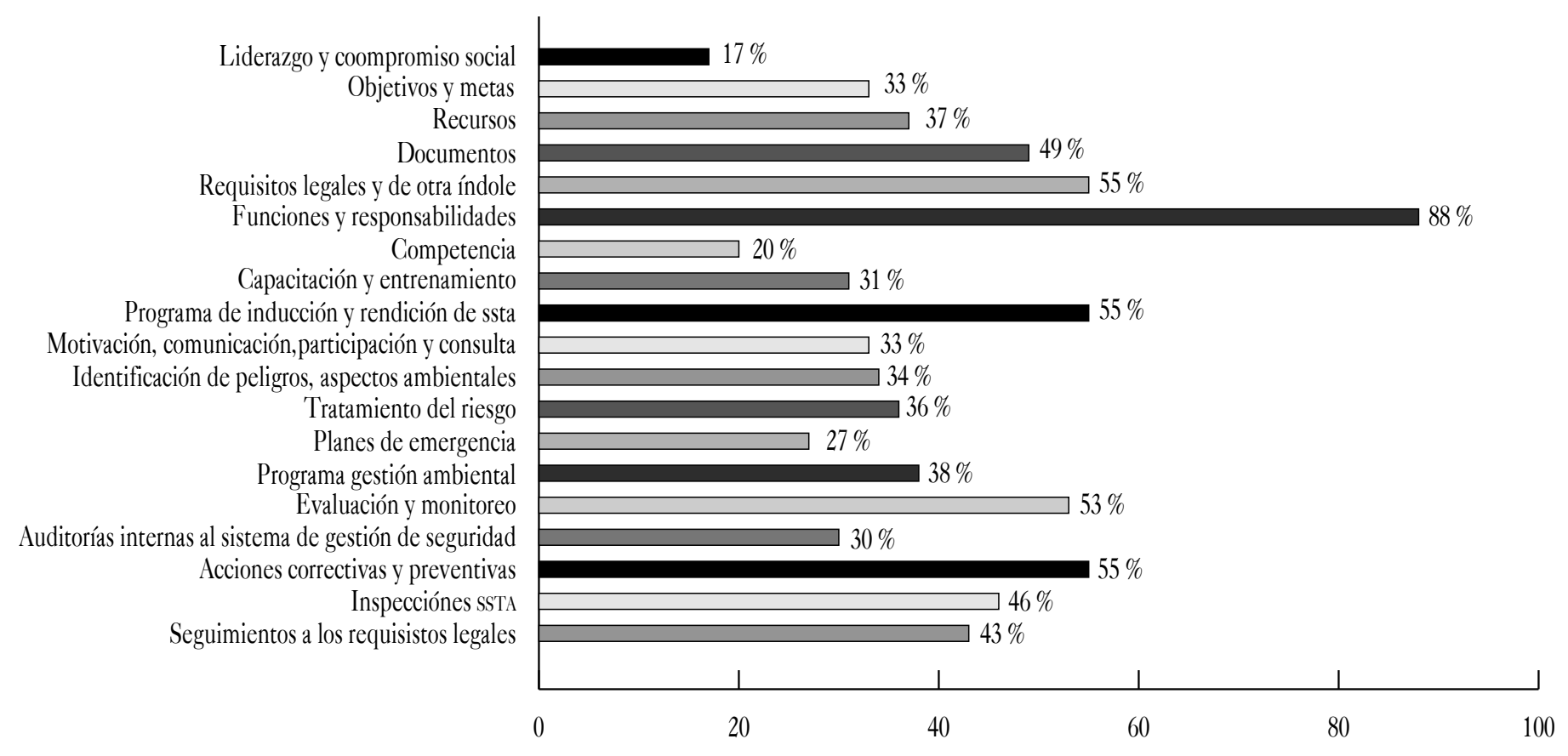

Fuente: elaboración propia.

La organización de la información se basó en un análisis sistemático y exhaustivo de los riesgos vinculados a esta actividad. Además de ayudar a poner de relieve y evaluar los distintos riesgos que puedan estar relacionados con este sector, el análisis y priorización de los factores de riesgo permite disponer de una estructura uniforme para la rápida familiarización y fácil orientación, así como el empleo de términos y expresiones normalizados para toda la gama de riesgos referidos, lo que favorece un reconocimiento instantáneo de los riesgos similares vinculados a esta actividad.

Con base en lo anterior, se priorizaron los riesgos mediante la aplicación de la GTC 45 (Icontec, 2012), y se elaboró la matriz para la identificación de peligros y valoración de riesgos de acuerdo con lo solicitado en el anexo técnico de esta norma. Frente a dicho análisis, los riesgos valorados con mayor relevancia (alto y muy alto) son tránsito, en actividades de conducción y mensajería; psicosocial, en actividades administrativas y de conducción; químico, en actividades de mantenimiento; mecánico, en actividades de mantenimiento; trabajo en alturas, en actividades de mantenimiento y conducción.

Además, se realizó un análisis de causas frente a uno de los riesgos con mayor relevancia en el grupo empresarial de Boyacá. 
Figura 2. Factores que inciden en la accidentalidad en trasporte de carga seca

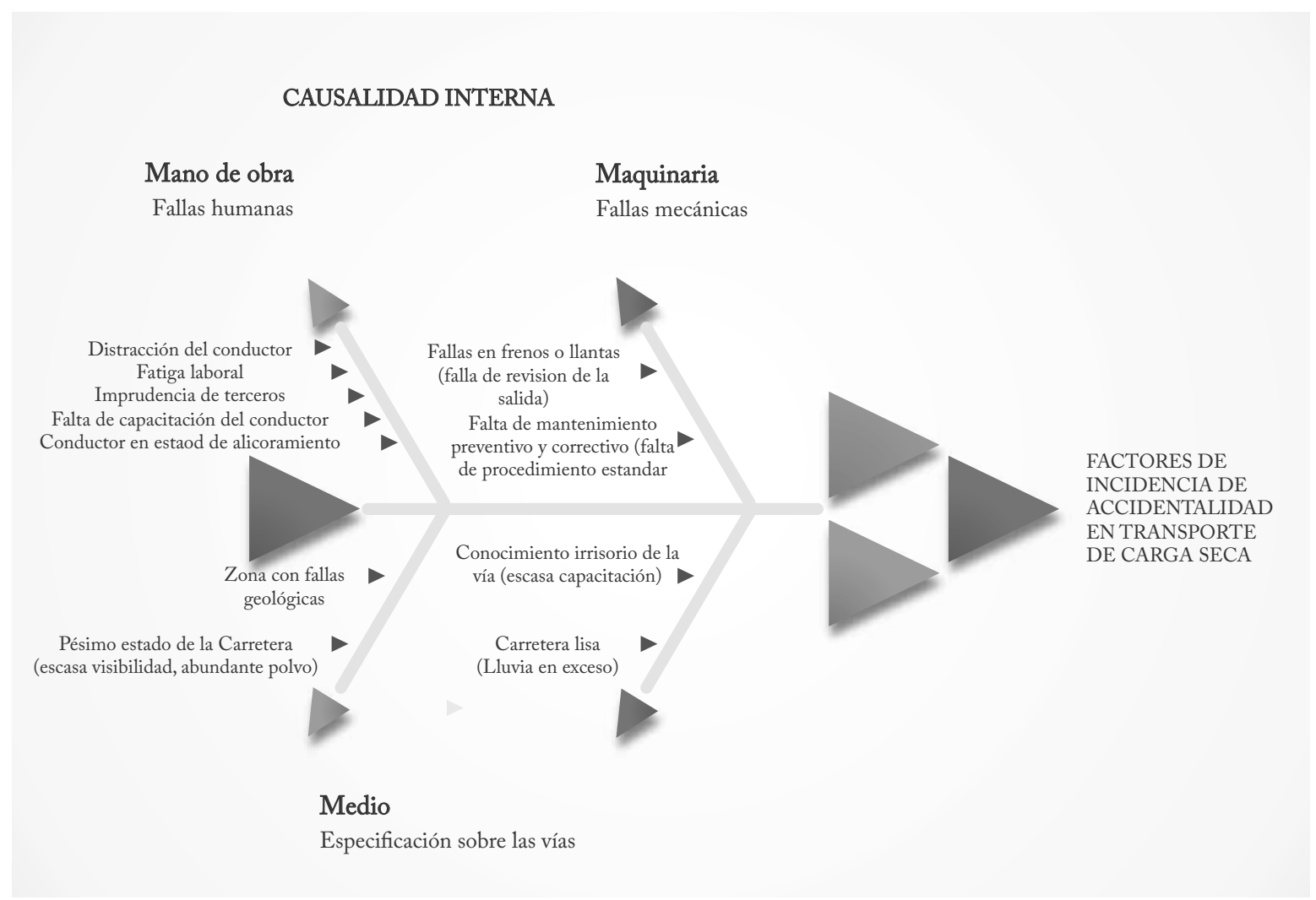

Fuente: elaboración propia.

Tabla 1. Planes y programas priorizados

\begin{tabular}{|l|l|l|l|l|}
\hline \multirow{2}{*}{ Tipo de riesgo } & \multicolumn{4}{|c|}{ Cargos } \\
\cline { 2 - 5 } & Administrativos & \multicolumn{1}{c|}{ Mensajeros } & \multicolumn{1}{c|}{ Conductores } & \multicolumn{1}{c|}{$\begin{array}{c}\text { Mecánicos - } \\
\text { pintores }\end{array}$} \\
\hline Tránsito & & $\begin{array}{l}\text { Plan estratégico de } \\
\text { seguridad vial }\end{array}$ & $\begin{array}{l}\text { Plan estratégico de } \\
\text { seguridad vial }\end{array}$ & \\
\hline $\begin{array}{l}\text { Químico } \\
\text { (PRQ) }\end{array}$ & & & $\begin{array}{l}\text { Programa de riesgo } \\
\text { químico }\end{array}$ \\
\hline $\begin{array}{l}\text { Trabajo en alturas } \\
\text { (PTA) }\end{array}$ & & & $\begin{array}{l}\text { Programa para el trabajo en } \\
\text { alturas }\end{array}$ & $\begin{array}{l}\text { Programa para el } \\
\text { trabajo en alturas }\end{array}$ \\
\hline $\begin{array}{l}\text { Psicosocial } \\
\text { (PRP) }\end{array}$ & Programa de riesgo psicosocial & & $\begin{array}{l}\text { Programa de riesgo } \\
\text { psicosocial }\end{array}$ & \\
\hline
\end{tabular}

Fuente: elaboración propia. 
Con base en la valoración de los factores clave y variables del modelo tomados de la fase anterior, se identifican los planes y programas que requieren evaluación prioritaria, como se presenta en la tabla 1.

Con base en los resultados de la valoración de los riesgos, se determina que esta información es prioritaria en la inclusión de criterios de auditoría en programa y plan de auditoría; en esta se identifican las siguientes variables:

- Proceso de auditoría (Iso 19011)

- Criterio de auditoría (resultados de la valoración de riesgos)
- Auditor competente (habilidades, formación, experiencia, educación)

La evaluación inicial y continua de los auditores y auditores líderes debe estar planificada, implementada y registrada de acuerdo con los procedimientos del programa de auditoría para proporcionar un resultado que sea objetivo, coherente, veraz y fiable. El proceso de evaluación debe identificar las necesidades de formación y de mejora de otras habilidades.

A continuación, se ilustra la relación entre la evaluación inicial de un auditor, la selección del equipo auditor, el desarrollo profesional continuado, el mantenimiento de

Fiǵura 3. Etapas de la evaluación del auditor

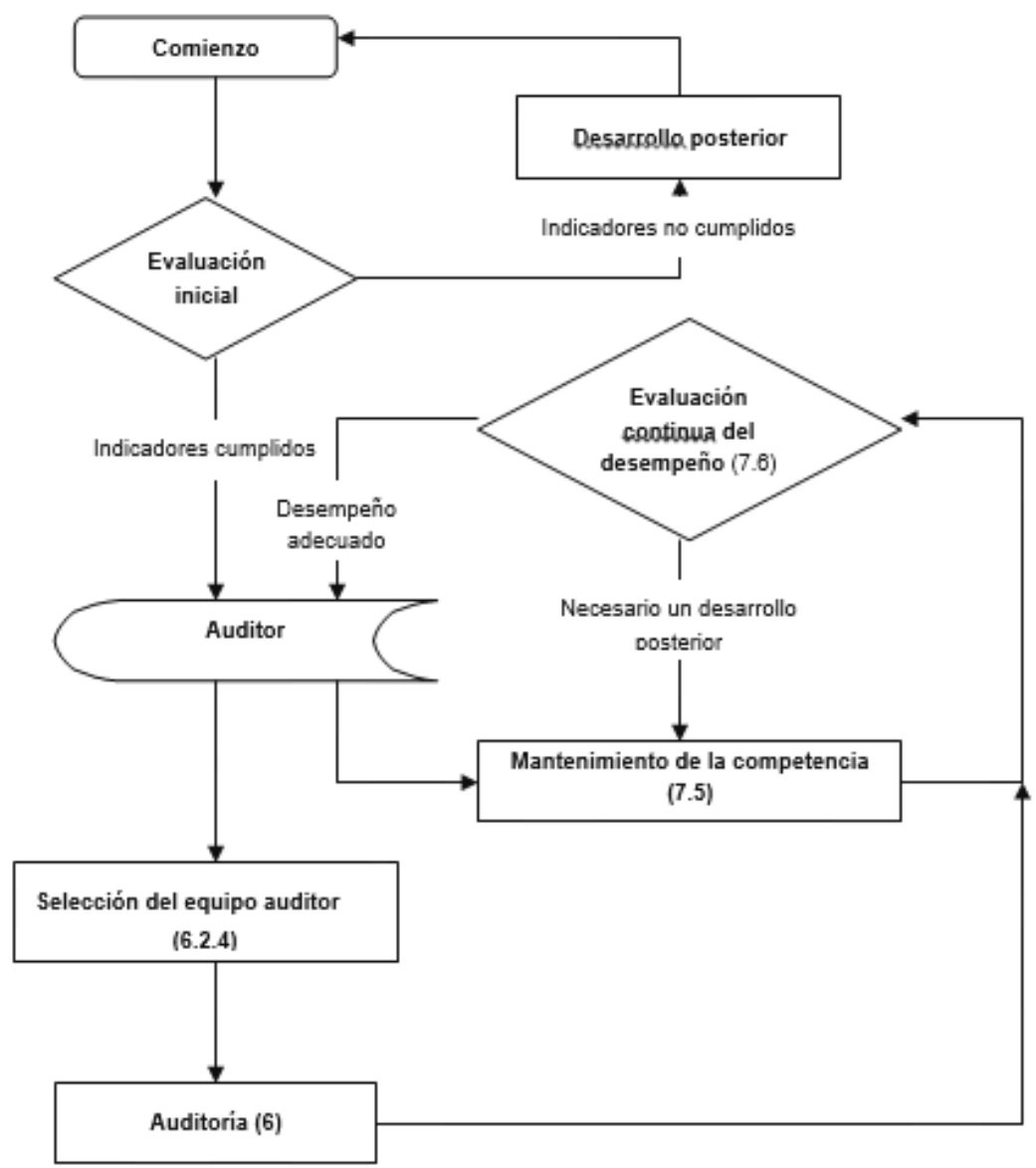

Fuente: elaboración propia. 
la capacidad de auditar y el proceso continuo de evaluación del auditor.

Finalizado el proceso de implementación de dichos programas y planes, así como su priorización según valoración, se procede a realizar auditorías internas con base en la aplicación de la norma NTC 19011 y el procedimiento de auditorías internas de la organización que incluye: programa de auditorías, plan de auditorías, listas de verificación, modelo de informe de auditoría. Además, por cada plan o programa evaluado se identifican los tipos de hallazgos de incumplimiento y se monitorea la aplicación del procedimiento de auditorías internas existente, para lo cual se asocian los hallazgos de la auditoría a cada programa, y estos a su vez se relacionan con los eventos de los resultados de la aplicación del procedimiento de la auditoría interna mediante la matriz de causalidad.

\section{Evaluación de competencia del auditor}

Se establece el análisis de la competencia del auditor con base en criterios definidos en Iso 19011, así: B = Deficiente (0-40), R = Regular (41-70), M = Aceptable (71-100). Del análisis se identifica que, para el plan estratégico de seguridad vial, el auditor con el nivel más alto de competencia es el EA1 con $75 \%$.

Tabla 2. Competencia de los auditores para la evaluación del plan estratégico de seguridad vial

\begin{tabular}{|c|c|c|c|c|c|}
\hline Plan Estrategico de Seguridad Vial (PESV) & \multicolumn{5}{|c|}{ Matriz de correlación de competencia del auditor } \\
\hline \multirow{2}{*}{ Competecia } & \multicolumn{5}{|c|}{ Auditor } \\
\hline & EA1 & EA2 & EA3 & EA4 & promedio \\
\hline Atributos personales & 90 & 50 & 70 & 60 & 67,5 \\
\hline Habilidades generales & 90 & 90 & 60 & 50 & 72,5 \\
\hline Aplicación de conocimientos y habilidades de los lideres de los equipos auditores & 80 & 70 & 40 & 80 & 67,5 \\
\hline Aplicación de principios y proceso de auditoría & 70 & 40 & 50 & 55 & 53,75 \\
\hline Manejo del tiempo de auditoría & 80 & 60 & 70 & 70 & 70 \\
\hline Aplicación de conocimiento de alcance y los criterios de auditoría & 80 & 70 & 80 & 75 & 76,25 \\
\hline Aplicación de conocimiento del Sistema de Gestión: SG-SST, OHSAS - RUC & 50 & 80 & 50 & 60 & 60 \\
\hline Conocimento en legislación & 70 & 60 & 70 & 40 & 60 \\
\hline Aplicación de conocimiento del contexto de las operaciones de la organización & 60 & 80 & 70 & 30 & 60 \\
\hline Nivel de estudios & 80 & 80 & 50 & 50 & 65 \\
\hline Experiencia en auditorías & 80 & 70 & 90 & 60 & 75 \\
\hline Total promedio & 75 & 68 & 64 & 57 & 66 \\
\hline
\end{tabular}

Fuente: elaboración propia.

Del presente análisis se identifica que, para el programa de riesgo químico, el auditor con el nivel más alto de competencia es el EA3 con $77 \%$. 
Tabla 3. Competencia de los auditores para la evaluación del programa de riesgo químico

\begin{tabular}{|l|c|c|c|c|c|}
\hline \multirow{2}{*}{ Programa de Riesgó Químico (PRQ) } & \multicolumn{5}{|c|}{ Matriz de correlación de competencia del auditor } \\
\hline \multirow{2}{*}{ Competencia } & \multicolumn{5}{|c|}{ Auditor } \\
\cline { 2 - 7 } & EA1 & EA2 & EA3 & EA4 & promedio \\
\hline Atributos personales & 50 & 50 & 70 & 80 & 62,5 \\
\hline Habilidades generales & 70 & 90 & 60 & 80 & 75 \\
\hline Aplicación de conocimientos y habilidades de los líderes de los equipos auditores & 40 & 70 & 80 & 80 & 67,5 \\
\hline Aplicación de principios y proceso de auditoría & 70 & 40 & 90 & 55 & 63,75 \\
\hline Manejo del tiempo de auditoría & 80 & 60 & 70 & 70 & 70 \\
\hline Aplicación de conocimiento de alcance y los criterios de auditoría & 60 & 70 & 80 & 75 & 71,25 \\
\hline Aplicación de conocimiento del Sistema de Gestión: SG-SST, oHSAS - RUC & 50 & 80 & 90 & 60 & 70 \\
\hline Conocimento en legislación & 70 & 60 & 70 & 70 & 67,5 \\
\hline Aplicación de conocimiento del contexto de las operaciones de la organización & 60 & 80 & 70 & 65 & 68,75 \\
\hline Nivel de estudios & 30 & 80 & 80 & 50 & 60 \\
\hline Experiencia en auditorías & 80 & 70 & 90 & 60 & 75 \\
\hline Total, promedio & 60 & 68 & 77 & 68 & 68 \\
\hline
\end{tabular}

Fuente: elaboración propia.

Del presente análisis se identifica que, para el programa de trabajo en alturas, el auditor con el nivel más alto de competencia es el EA1 con $75 \%$.

Tabla 4. Competencia de los auditores para la evaluación del programa de trabajo en alturas

\begin{tabular}{|l|c|c|c|c|c|}
\hline \multirow{2}{*}{ Progirama para el Trabajo en Alturas (PTA) } & \multicolumn{4}{|c|}{ Matriz de correlación de competencia del auditor } \\
\hline \multirow{2}{*}{ Competencia } & \multicolumn{4}{|c|}{ Auditor } \\
\cline { 2 - 7 } & EA1 & EA2 & EA3 & EA4 & \multicolumn{2}{c|}{ promedio } \\
\hline Atributos personales & 90 & 50 & 70 & 60 & 67,5 \\
\hline Habilidades generales & 50 & 90 & 60 & 50 & 62,5 \\
\hline Aplicación de conocimientos y habilidades de los líderes de los equipos auditores & 80 & 70 & 40 & 90 & 70 \\
\hline Aplicación de principios y proceso de auditoría & 70 & 40 & 50 & 55 & 53,75 \\
\hline Manejo del tiempo de auditoría & 80 & 60 & 70 & 50 & 65 \\
\hline Aplicación de conocimiento de alcance y los criterios de auditoría & 80 & 70 & 80 & 50 & 70 \\
\hline Aplicación de conocimiento del Sistema de Gestión: SG-SST, oHSAS-RUC & 90 & 80 & 50 & 60 & 70 \\
\hline Conocimento en legislación & 70 & 60 & 70 & 40 & 60 \\
\hline Aplicación de conocimiento del contexto de las operaciones de la organización & 60 & 80 & 40 & 30 & 52,5 \\
\hline Nivel de estudios & 80 & 80 & 50 & 50 & 65 \\
\hline Experiencia en auditorías & 80 & 70 & 90 & 60 & 75 \\
\hline Total, promedio & $\mathbf{7 5}$ & 68 & 61 & $\mathbf{5 4}$ & $\mathbf{6 5}$ \\
\hline
\end{tabular}

Fuente: elaboración propia. 
Del presente análisis se identifica que, para el programa de riesgo psicosocial, los auditores con el nivel más alto de competencia son EA1 y EA2 con 75\% y 76\%.

Tabla 5. Competencia de los auditores para la evaluación del programa de riesgo psicosocial

\begin{tabular}{|l|c|c|c|c|c|}
\hline \multirow{2}{*}{ Programa de Riesgo Psicosocial (PRPS) } & \multicolumn{4}{|c|}{ Matriz de correlación de competencia del auditor } \\
\cline { 2 - 7 } Competencia & EA1 & EA2 & EA3 & EA4 & \multicolumn{2}{|c|}{ promedio } \\
\hline Atributos personales & 90 & 90 & 70 & 60 & 77,5 \\
\hline Habilidades generales & 50 & 90 & 60 & 50 & 62,5 \\
\hline Aplicación de conocimientos y habilidades de los líderes de los equipos auditores & 80 & 80 & 50 & 90 & 75 \\
\hline Aplicación de principios y proceso de auditoría & 70 & 80 & 50 & 55 & 63,75 \\
\hline Manejo del tiempo auditoría & 80 & 60 & 70 & 50 & 65 \\
\hline Aplicación de conocimiento de alcance y los criterios de auditoría & 80 & 70 & 80 & 50 & 70 \\
\hline Aplicación de conocimiento del Sistema de Gestión: SG-SST, OHSAS - RUC & 90 & 80 & 50 & 60 & 70 \\
\hline Conocimento en legislación & 70 & 60 & 70 & 40 & 60 \\
\hline Aplicación de conocimiento del contexto de las operaciones de la organización & 60 & 80 & 50 & 30 & 55 \\
\hline Nivel de estudios & 80 & 80 & 50 & 50 & 65 \\
\hline Experiencia en auditorías & 80 & 70 & 90 & 60 & 75 \\
\hline Total, promedio & 75 & 76 & 63 & $\mathbf{5 4}$ & 67 \\
\hline
\end{tabular}

Fuente: elaboración propia.

\section{Diseño de modelo de auditorías de valor}

Con base en los resultados de las auditorías, a cada uno de los programas establecidos y los resultados de la evaluación del desarrollo de la aplicación de la auditoría interna, en consideración con las variables identificadas como relevantes, se simula (aplicando dinámica de sistemas Forrester) el comportamiento de un modelo de gestión de auditorías de valor que incorpore las herramientas necesarias para evaluar la eficacia del sistema de gestión con base en la OHSAS 18001:2007 y RUC, con el propósito de mejorar la evaluación de los riesgos que intervienen en la aparición de accidentes.

En entrevista con expertos en auditoría, auditores de certificación y consultores de sistemas de gestión, y de acuerdo con la evaluación realizada a la ejecución de auditoría de primera parte, llevadas a cabo por auditores de tercera parte en procesos de certificación, se observa que dichas auditoría de primera parte (auditorías internas) no generan resultados que le aporten a las empresas de transporte. Este fenómeno repercute en las estadísticas de accidentalidad y el incremento de peticiones, quejas, reclamos y sugerencias en el grupo empresarial analizado. Lo anterior se confirma con la opinión y la percepción de dichos expertos sobre la necesidad de mejorar el enfoque de las auditorías internas.

Posteriormente, se diseña un modelo de gestión de auditorías internas de valor que permite el aprovechamiento de esta herramienta de evaluación como fuente importante para la identificación de necesidades de mejora en la 
planificación, ejecución y seguimiento en la realización de auditorías internas, teniendo en cuenta los riesgos más relevantes en la empresa.

A través del diagnóstico y la implementación del SG-SST, se han identificado algunos problemas desde su implementación:

- Los auditores: no se cuenta con la competencia necesaria para llevar a cabo el proceso.

- Los procesos de certificación: se han visto las fallas recurrentes en los informes de auditoría interna, donde los resultados no aportan hallazgos que le aporten al mejoramiento en el desempeño de sus procesos.
Esto puede tener múltiples razones. En esta investigación se trata de hallar, analizar y comprender sus funcionamientos respecto a todo el sistema.

\section{Diagrama causal}

El conjunto de los elementos que tienen relación con el presente problema permite en principio explicar los procesos de auditoría observados, junto con las relaciones entre empresa-auditor-auditado y el escenario en la ejecución de la auditoría. El diagrama causal que aquíse presenta (figura 4) es un modelo que recoge los elementos clave del proceso de auditoría interna y las relaciones entre sus variables.

Fiǵura 4. Relación causa-efecto del objeto del estudio

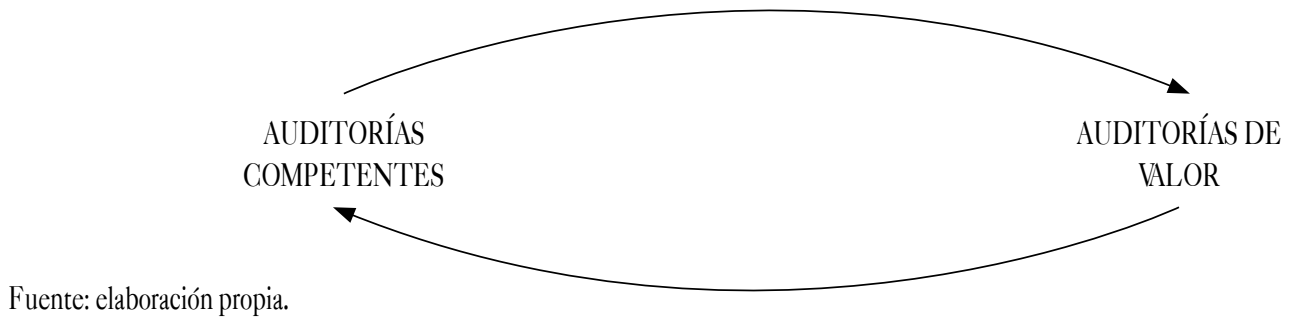

En la figura 4 se puede evidenciar que uno de los principales problemas para la implementación del SG-SST son los auditores, que no cuentan con la competencia necesaria para llevar a cabo el proceso de auditoría; una prueba de ello son las fallas recurrentes en los informes de auditoría interna, en los que los resultados no aportan hallazgos significativos que generen un mejoramiento en el desempeño de los procesos.

Figura 5. Relación de retroalimentación entre los componentes del sistema

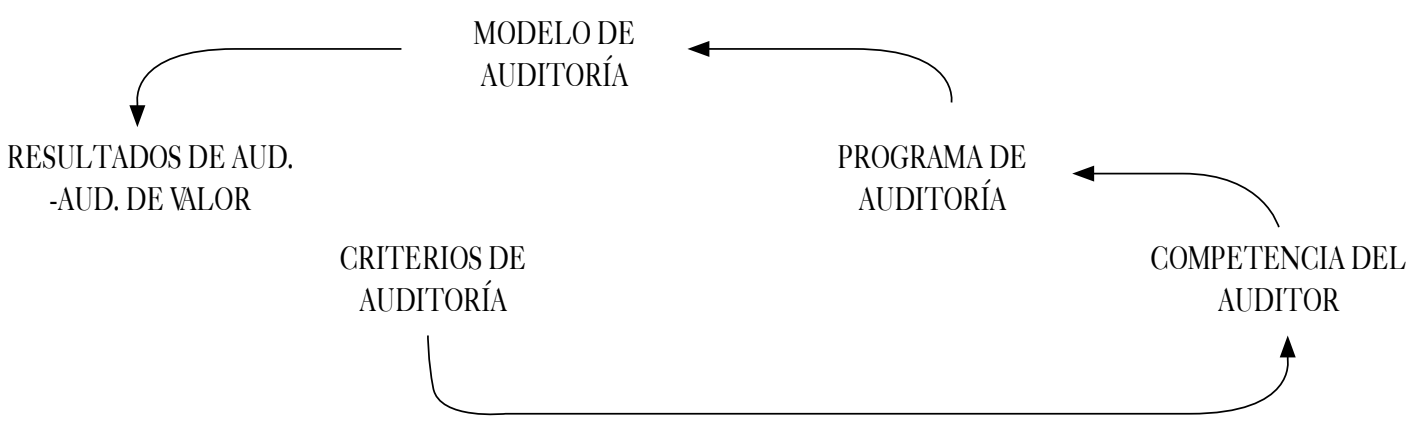

Fuente: elaboración propia. 
La gama mínima de elementos y relaciones ha permitido reproducir la referencia histórica, que permite formar la estructura básica del sistema. Una vez conocidas globalmente las variables del sistema y las hipotéticas relaciones causales existentes entre ellas, pasamos a la representación gráfica de la relación de la retroalimentación entre los componentes del sistema (figura 5).

Figura 6. Diagrama de causas y comportamiento de niveles de investigación

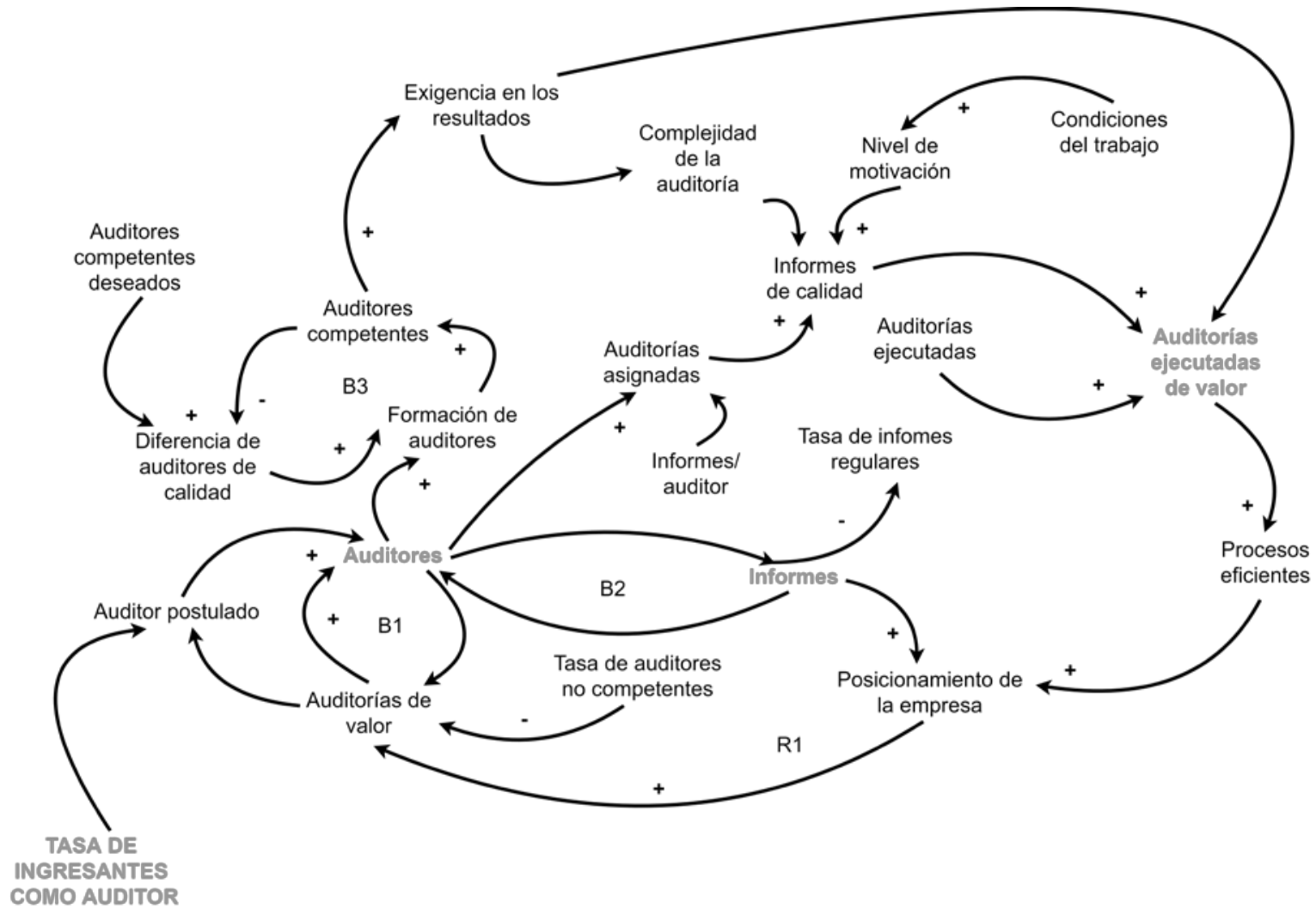

Fuente: elaboración propia.

En la figura 6, que expone las causas y comportamiento de niveles de investigación, se evidencia que cuando un auditor tiene mayor competencia se espera que los resultados sean mejores y su desempeño se vea acorde con empresas grandes y de mayor complejidad. Por lo tanto, el auditor competente hace que sus auditados estén más preparados para enfrentar el proceso de auditoría y no permite la identificación de hallazgos de incumplimiento por parte del auditor, por lo que se esperaría que dichos auditados tengan mayor competencia.
Pero la utilidad más importante de esta concepción es comprender cómo la estructura de los sistemas provoca su comportamiento. En una misma empresa y un mismo sistema de gestión (tomando el caso del grupo empresarial OIT) hay varios auditores que realizan auditorías y presentan resultados muy diferentes; por lo tanto, en realidad deberían estudiar por qué los sistemas que controlan sus empresas tienen unos resultados que no aportan a la real identificación de sus problemas estructurales, situación que conlleva a que sea menos 
competitiva que en otros escenarios de evaluación que han presentado mejores resultados.

Finalmente, se diseña el Modelo de Gestión de Auditorías de valor GI-PD-008, documento que establece cómo evaluar el avance al proceso de implementación del sistema de gestión, incluyendo el plan estratégico de seguridad vial. El concepto auditoría de valor nos plantea que el sistema de gestión de la empresa es un reflejo real de lo que tiene, y que mientras más experiencia tiene el auditor, este logra identificar los hallazgos; de esta manera, el juicio de valor es que el auditor sea competente para entender la situación real de la empresa. Comparando lo bueno y lo malo de la salida, la auditoría muestra la situación real de la empresa, para que se identifiquen los hallazgos que la organización realmente necesita para poder establecer acciones correctivas de manera eficaz.

El modelo de auditoría de valor inicia con la abstracción de las variables que permiten entender el problema, es decir, una abstracción de la realidad. El análisis causal permite construir un modelo que muestra la relación del análisis de causas y las tablas históricas para tratar de entender el funcionamiento de un proceso de auditoría. Por su complejidad, se lleva a cabo una simulación que valide y pueda reproducir la realidad con un análisis estadístico histórico. Este modelo se asocia a un modelo de tipo probabilístico, de modo que se modela el problema para el análisis de causa, tratando de entender el modelo para comprender la interacción en los escenarios relevantes en un escenario de auditoría. Este modelo permite garantizar la estructura para realizar una buena auditoría, con valores agregados, y que permita minimizar los efectos de variables que pueden afectar su calidad.

Para validar el modelo de la figura 7 se propone realizar una simulación en tiempo discreto, que busca reproducir el comportamiento de las tablas históricas y que muestre que las variables expuestas en el modelo anterior sí afectan la calidad de la auditoría interna. La muestra tomada para la realización de los históricos corresponde a 69 empresas de diferentes sectores y tamaños, que han permitido el análisis de las tablas de probabilidad y que resume las frecuencias de las variables registradas en las auditorías, para lo cual se plantea el modelo de simulación aplicando herramientas estadísticas y Promodel.

Este modelo sintetiza las diferentes etapas de los procesos de auditoría, que se presentan en el análisis de la realidad. Los planteamientos de los modelos de gestión como el taylorismo obedecen a algunas etapas adoptadas en el modelo de auditoría de valor, para representar el aporte en la gestión de auditorías de valor, teniendo en cuenta diferentes etapas como la observación, la experimentación, la inducción, el análisis y la síntesis. 
Carlos Alirio Beltrán Rodríguez, Ángel Leonel Puerto Núñez, Harold Wilson Hernández Cruz

Figura 7. Modelo propuesto para la generación de auditorías de valor

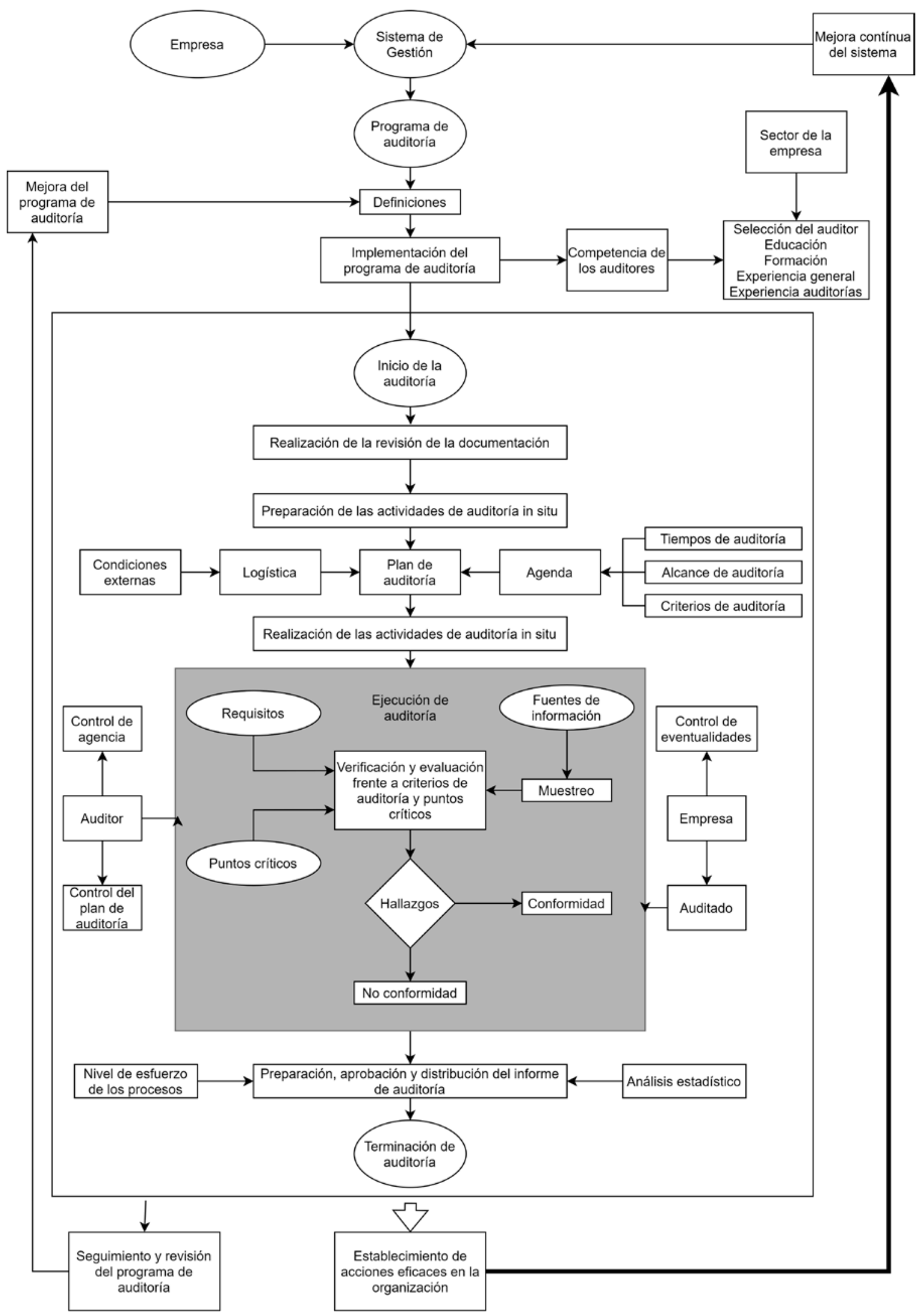

Fuente: elaboración propia. 


\section{CONCLUSIONES}

La competencia del personal encargado de llevar a cabo las auditorías es fundamental, ya que se busca evitar la subjetividad en la obtención de hallazgos. Se ratifica que los hallazgos derivados de un proceso de auditoría deben ser oportunidades de mejora y no conformidades que conlleven a la organización a realizar un buen análisis de causas y, a la vez, a establecer planes de acción y mecanismos para la evaluación de la eficacia de las acciones, de tal modo que se garantice la no recurrencia de los problemas.

Además, se plantea la realización de procesos de formación al personal sobre la nueva metodología y formación específica en la GTC ISO 19011, para adquirir las habilidades necesarias para la ejecución de auditorías con el concepto de auditorías de valor.

Finalmente, este proyecto se constituye como un aporte al grupo empresarial abordado, en la identificación de su estado frente al cumplimiento de requisitos legales y reglamentarios, así como en la incorporación de metodologías de mejoramiento para la implementación de las normas OHSAS 18001:2007, la guía RUC y el plan estratégico de seguridad vial.

\section{REFERENCIAS}

Agencia Nacional de Seguridad Vial. (2019). Boletín estadístico de Boyacá. Fallecidos y lesionados. Recuperado de https://ansv.gov.co/observatorio/public/documentos/Boletin_Boyac\%C3\%A1_may_2019.pdf

Icontec. (2012). GTC 45. Guía para la identificación de los peligros y la valoración de los riesgos en seguridady salud ocupacional. Icontec.

Ministerio del Trabajo. (2015). Decreto 1072 de 2015, por medio del cual se expide el Decreto Único Reglamentario del Sector Trabajo. Recuperado de http://www. mintrabajo.gov.co/normatividad/decreto-unicoreglamentario

Ministerio de Transporte. (2014). Resolución 1565 de 2015, por la cual se expide la Guía metodológica para la elaboración del Plan Estratégico de Seguridad Vial. Recuperado de https://www.arlsura.com/files/ res1565_14.pdf

Robson, L. S., Clarke, J. A., Cullen, K., Bielecky, A., Severin, C., Bigelow, P. L., ... Mahood, Q. (2007). The effectiveness of occupational health and safety management system interventions: A systematic review. Safety Science, 45(3), 329-353. DoI: https://doi. org/10.1016/j.ssci.2006.07.003

Robson, L. S., Macdonald, S., Gray, G. C., Van Eerd, D. L., \& Bigelow, P. L. (2012). A descriptive study of the OHS management auditing methods used by public sector organizations conducting audits of workplaces: Implications for audit reliability and validity. Safety Science, 50(2), 181-189. DoI: https:// doi.org/10.1016/j.ssci.2011.08.006 